\title{
负载二甲酸钾缓释抗菌微球的构建
}

\author{
郭小炜 ${ }^{1}$, 李玉妍 ${ }^{1}$, 陈南春 ${ }^{1}$, 王秀丽 ${ }^{2}$, 解庆林 ${ }^{3}$ \\ (1. 桂林理工大学 材料科学与工程学院, 桂林 541004; 2. 桂林理工大学 化学与生物工程学院, 桂林 541006; \\ 3. 桂林理工大学 广西岩溶地区水污染控制与用水安全保障协同创新中心, 桂林 541006) \\ 摘 要: 为避免二甲酸钾 $(\mathrm{KDF})$ 在酸性环境下分解过快, 调节仔猪肠胃道酸碱性和菌落平衡, 实现 KDF 靶向释放 \\ 抗菌, 本研究以可生物降解的壳聚糖(CS)、羧甲基纤维素 $(\mathrm{CMC})$ 和无机刚性材料 $\mathrm{P}$ 型沸石分子篎(Zeolite P)为载体, \\ 负载抗菌药物二甲酸钾制备控释水凝胶微球。发现 $\mathrm{CS}$ 中 $-\mathrm{NH}_{2}$ 与 $\mathrm{CMC}$ 中- $\mathrm{COOH}$ 离子作用, 可形成结构稳定的聚 \\ 电解质复合物。溶胀率的差异性表明 $\mathrm{CS} / \mathrm{CMC} /$ Zeolite $\mathrm{P}$ 水凝胶微球对 $\mathrm{pH}$ 高度敏感。加入 Zeolite $\mathrm{P}$ 使水凝胶微球 \\ 在 $\mathrm{pH} 1.2$ 保持原有形貌且不被降解破裂。CS/CMC/Zeolite P/KDF 抗菌微球的包封率为 $47.75 \%$, 载药率为 $23.88 \%$, 可 \\ 有效缓释 $\mathrm{KDF}$, 在 $\mathrm{pH} 7.4$ 磷酸盐缓冲溶液中的缓释性比 $\mathrm{pH} 1.2$ 更优。 $\mathrm{CS} / \mathrm{CMC} /$ Zeolite $\mathrm{P} / \mathrm{KDF}$ 抗菌微球浓度为 $96 \mathrm{mg} / \mathrm{mL}$ \\ 时对大肠杆菌最大抑菌率为 $83 \%$, 有效提高了 $\mathrm{KDF}$ 利用率。
}

关 键 词: 二甲酸钾; 水凝胶微球; 缓释; 抗菌

中图分类号: TB332 文献标识码: A

\section{Construction of Sustainable Release Antimicrobial Microspheres Loaded with Potassium Diformate}

\author{
GUO Xiaowei ${ }^{1}$, LI Yuyan ${ }^{1}$, CHEN Nanchun ${ }^{1}$, WANG Xiuli ${ }^{2}$, XIE Qinglin ${ }^{2}$
}

(1. School of Materials Science and Engineering, Guilin University of Technology, Guilin 541004, China; 2. School of Chemical and Biological Engineering, Guilin University of Technology, Guilin 541006, China; 3. Guangxi Collaborative Innovation Center for Water Pollution Control and Water Safety in Karst Area, Guilin University of Technology, Guilin 541006, China)

\begin{abstract}
To prevent potassium diformate (KDF) from decomposing too quickly in an acidic environment, adjust the acidity and alkalinity of the gastrointestinal tract of the piglets and the colony balance, and realize the targeted release of KDF antibacterial, in this study, a kind of hydrogel microspheres were prepared by biodegradable chitosan (CS), carboxymethyl cellulose (CMC), and inorganic rigid material P-type molecular sieve (Zeolite P). Then the antibacterial drug potassium diformate was loaded so as to be controlled to release from this hydrogel microsphere. The results show that $-\mathrm{NH}_{2}$ in $\mathrm{CS}$ and $-\mathrm{COOH}$ in $\mathrm{CMC}$ form a stable polyelectrolyte complex through ion interaction. Swelling rate test indicates that the $\mathrm{CS} / \mathrm{CMC} /$ Zeolite $\mathrm{P}$ hydrogel microspheres display high $\mathrm{pH}$-sensitivity. The addition of Zeolite $\mathrm{P}$ allows the hydrogel microspheres to maintain their original morphology at $\mathrm{pH} 1.2$ without being degraded or broken. The effective sustained-release KDF in $\mathrm{pH} 7.4$ phosphate buffer solution exhibits better sustained-release performance
\end{abstract}

收稿日期: 2020-03-12; 收到修改稿日期：2020-05-20; 网络出版日期: 2020-06-09

基金项目 : 国家自然科学基金(51564008，41662005); 广西科技攻关项目(11107003-4); 广西自然科学基金(2015GXNSFAA139094, 2016GXNSFAA380014)

National Natural Science Foundation of China (51564008, 41662005); Guangxi Science and Technology Project (11107003-4); Natural Science Foundation of Guangxi (2015GXNSFAA139094, 2016GXNSFAA380014)

作者简介: 郭小炜(1993-), 男, 硕士研究生. E-mail: 781860301@qq.com GUO Xiaowei(1993-), male, Master candidate. E-mail: 781860301@qq.com

通信作者：陈南春，教授.E-mail: cnc@glut.edu.cn; 王秀丽，高级工程师.E-mail:610064853@qq.com CHEN Nanchun, professor. E-mail: cnc@glut.edu.cn; WANG Xiuli, senior engineer. E-mail: 610064853@qq.com 
than that in $\mathrm{pH} 1.2$. When the concentration of $\mathrm{CS} / \mathrm{CMC} /$ Zeolite $\mathrm{P} / \mathrm{KDF}$ antimicrobial microspheres is $96 \mathrm{mg} / \mathrm{mL}$, the maximum bacteriostatic rate of Escherichia coli is $83 \%$, which effectively improves the utilization rate of KDF.

Key words: potassium diformate; hydrogel microsphere; sustained-release; antibacterial

抗生素自 20 世纪 50 年代应用于养殖业以来, 极大地促进了养殖业的快速发展, 但带来的药物残 留 ${ }^{[1]}$ 和耐药性 ${ }^{[2]}$ 问题越来越严重。二甲酸钾 $(\mathrm{KDF})^{[3]}$ 是 2001 年被欧盟批准使用的第一种替代抗生素促 生长剂的非抗生素饲料添加剂, 近年来开发和应用 速度较快。KDF 添加到动物饲料中可平衡动物胃肠 道 $\mathrm{pH}$ 、调节肠道微生态平衡、提高饲料营养物质的 消化和吸收等 ${ }^{[4-7]}$ 。但直接喂食 $\mathrm{KDF}$ 药物的利用率 不高, 主要在小肠前端起作用, 不能充分发挥 KDF 调节肠道菌群的功能。

近年来, 利用 $\mathrm{KDF}$ 制备抗菌剂的研究有了研究 进展。张小虎等 ${ }^{[8]}$ 利用 $\mathrm{A}$ 型沸石分子篮在常温下直 接吸附 $\mathrm{KDF}$ ，制备抗菌剂，抗菌期达到 2 3 d。 $\mathrm{Hu}$ 等 ${ }^{[9]}$ 利用 $\mathrm{X}$ 型沸石分子篮, 以 $\mathrm{CS}$ 为中间体复配 $\mathrm{KDF}$ 制备缓释抗菌剂, $\mathrm{X}$ 型沸石分子篎、 $\mathrm{CS}$ 和 $\mathrm{KDF}$ 可以形成有效的接枝，体外抑菌率可达 $78.16 \%$ 。但 这些进展仍没有充分提高 KDF 的利用效率。

羧甲基纤维素 $(\mathrm{CMC})^{[10]}$ 是一种阴离子性高聚物, 每条分子链上有 3 个游离羟基。这些游离羟基决定 了 $\mathrm{CMC}$ 在水溶液中能与带正电荷的基团结合。壳 聚糖 $(\mathrm{CS})^{[11]}$ 是带正电荷的天然高分子, 其分子链上 存在大量氨基基团。 $\mathrm{CMC}$ 和 $\mathrm{CS}$ 两者具有 $\mathrm{pH}$ 敏感 性 ${ }^{[12-13]}$, 可使两种高分子在水溶液中交联, 形成聚 电解质复合物。在其中引入无机刚性材料可提高机 械强度和稳定性 ${ }^{[14]}$ 。在常用的缓释剂无机材料中, 分子筛因具有优异的生物相容性、低毒性、突出的 热稳定性和力学性能而受到极大的关注, 并已应用 于污水处理、生物医药和畜牧业生产中 ${ }^{[15-17]}$ 。分子 篮还具有极大的比表面积和较强的吸附能力, 能有 效地提高药物负载量, 既可与药物结合, 又具有缓 释效应。

本研究使用 $\mathrm{CaCl}_{2}$ 作为螯合剂, 将 $\mathrm{CS} 、 \mathrm{CMC}$ 和 Zeolite $\mathrm{P}$ 通过静电自组装制备成复合水凝胶微球 $\mathrm{CS} / \mathrm{CMC} /$ Zeolite $\mathrm{P}$, 作为 $\mathrm{pH}$ 敏感的药物载体, 以 $\mathrm{KDF}$ 为模型药物, 制备水凝胶抗菌微球, 可使 $\mathrm{KDF}$ 在小肠中后段及大肠段发挥作用。

\section{1 实验方法}

\section{1 实验试剂}

实验所用的 $\mathrm{P}$ 型沸石分子篮为自制; 壳聚糖(脱
乙酰度 $>90.0 \%$, 化学纯)购自上海蓝季生物; 羧甲基 纤维素(黏度: $800 \sim 1200 \mathrm{mPa} \cdot \mathrm{s}$, 化学纯)购自国药 集团化学试剂有限公司; 无水氯化钙和乙酸为分析 纯, 购自西陇科学股份有限公司; 二甲酸钾(含量 $\geqslant$ 98\%)购自武汉远成科技有限公司。

\section{2 实验过程}

\subsection{1 药物载体的制备}

$\mathrm{P}$ 型沸石分子篮基于文献[18-19]报道的方法制 备。 $0.3 \mathrm{~g}$ Zeolite P 置于 $100 \mathrm{~mL}$ 去离子水中超声分 散 $10 \mathrm{~min}$, 缓慢加入 $3.0 \mathrm{~g} \mathrm{CMC}$, 边加入边缓慢搅拌, 形成均一溶液后静置 $12 \mathrm{~h}$, 得到溶液 $\mathrm{A}$ 。称取 $4 \mathrm{~g}$ $\mathrm{CaCl}_{2} 、 2 \mathrm{~g} \mathrm{CS}$ 溶解于 $100 \mathrm{~mL} 2 \%$ 醋酸溶液，静置 $12 \mathrm{~h}$, 得到混合溶液 $\mathrm{B}$ 。用 $5 \mathrm{~mL}$ 针筒缓慢把溶液 $\mathrm{A}$ 滴加到 溶液 B 中, 搅拌 $60 \mathrm{~min}$, 去离子水洗涤, 冷冻干燥, 即 可得到 $\mathrm{CS} / \mathrm{CMC} /$ Zeolite $\mathrm{P}$ 复合水凝胶微球。

\subsection{2 抗菌微球的制备}

Zeolite $\mathrm{P}(0.5 \mathrm{wt} \%$ 和 $1.5 \mathrm{wt} \%)$ 置于 $100 \mathrm{~mL}$ 去离子 水中超声分散 $10 \mathrm{~min}$, 加入 $0.2 \mathrm{~g} \mathrm{KDF}$, 摚拌 $3 \mathrm{~h}^{[20]}$, 再将 $3.0 \mathrm{~g} \mathrm{CMC}$ 缓慢加入 Zeolite P 与 KDF 混合溶 液, 边加入边缓慢摚拌, 形成均一溶液后静置 $12 \mathrm{~h}$, 得到溶液 $\mathrm{C}$ 。用 $5 \mathrm{~mL}$ 针筒缓慢把溶液 $\mathrm{C}$ 滴加到溶 液 $\mathrm{B}$ 中, 摚拌 $1 \mathrm{~h}$ 。过滤, 去离子水洗涤, 冷冻干燥, 即可得到 $\mathrm{CS} / \mathrm{CMC} /$ Zeolite $\mathrm{P} / \mathrm{KDF}$ 抗菌微球。制备过 程见图 1。

\section{3 结构表征与性能测试}

把 $\mathrm{CS} / \mathrm{CMC} /$ Zeolite $\mathrm{P}$ 复合水凝胶微球剖开, 用 扫描电子显微镜(SEM, 日本 JSM 6380-LV 型)观察样 品内部微观形貌。利用傅里叶变换红外光谱仪(FT-IR, 美国 Thermo Nicolet Nexus 470 型)分析材料官能团在 波数 $4000 \sim 450 \mathrm{~cm}^{-1}$ 之间的变化, 样品采用 $\mathrm{KBr}$

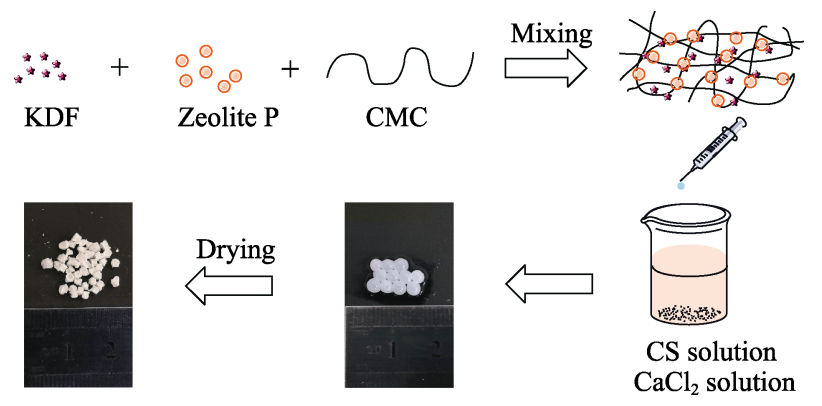

图 1 负载 $\mathrm{KDF}$ 的抗菌微球的制备过程

Fig. 1 Preparation of antibacterial microspheres loaded with KDF 
压片。用热重分析仪(美国 TA 公司 TGA Q500)对材 料进行热稳定性测试, 测试在大气氛围中进行, 升 温速率为 $10{ }^{\circ} \mathrm{C} / \mathrm{min}$ 。

\section{4 溶胀行为测试}

通过浸泡法测定水凝胶微球吸水性, 研究其 溶胀行为。配置 $\mathrm{pH} 1.2$ (模拟胃液), $\mathrm{pH} 6.8$ (模拟小肠 液), $\mathrm{pH} 7.4$ (模拟大肠液) 磷酸盐缓冲溶液, 冷藏待 用。分别称量 $\mathrm{CS} / \mathrm{CMC} /$ Zeolite $\mathrm{P}$ (Zeolite $\mathrm{P}$ 含量为 $0 、 0.5 \mathrm{wt} \%$ 和 $1.5 \mathrm{wt} \%) 0.02 \mathrm{~g}$ 干燥后的均匀水凝胶 微球颗粒, 浸泡在相应 $\mathrm{pH}$ 的磷酸盐缓冲溶液中。 在磁力搅拌器中 $500 \mathrm{r} / \mathrm{min}$ 缓慢搅拌, 在 $0.5 、 1 、 2 、$ $3 、 4 、 5 、 6 、 7 \mathrm{~h}$ 时间段分别取出微球, 用滤纸吸 附表面粘附的液体, 立即称重。通过公式(1)计算微 球的溶胀率:

$$
\text { Swelling }=\frac{M_{t}-M_{0}}{M_{0}} \times 100 \%
$$

式中, $M_{t}$ 为某一时间 $t$ 水凝胶微球吸水膨胀后的质 量, $M_{0}$ 为水凝胶微球初始质量。

\section{$1.5 \mathrm{KDF}$ 缓释行为分析}

称取一定质量的 $\mathrm{KCl}$, 用去离子水 $(\mathrm{pH}=7.4)$ 配制 浓度为 $0 、 5 、 10 、 15 、 20 、 25 、 30 \mathrm{mg} / \mathrm{L}$ 的钾离子标 准溶液, 用 novAA400p 型原子吸收分光光度计测定 各浓度的吸光度, 得到钾离子的标准曲线方程(2):

$$
y=0.03116 x+0.01507
$$

式中, $y$ 代表吸光度, $x$ 代表钾离子浓度 $(\mathrm{mg} / \mathrm{L})$, 拟合 相关系数 $R^{2}=0.99676$ 。

称取 $0.4 \mathrm{~g} \mathrm{CS} / \mathrm{CMC} /$ Zeolite $\mathrm{P} / \mathrm{KDF}$ 抗菌微球 (Zeolite P 含量分别为 $0 、 0.5 \mathrm{wt} \%$ 和 $1.5 \mathrm{wt} \%$ ) 置于 $100 \mathrm{~mL}$ 磷酸盐缓冲溶液中 $(\mathrm{pH}=7.4$, 不含钾离子 $)$, 在磁力 搅拌器中 $500 \mathrm{r} / \mathrm{min}$ 缓慢搅拌, 在 $15 \mathrm{~min} 、 30 \mathrm{~min} 、 1 \mathrm{~h}$ 、 $1.5 \mathrm{~h} 、 2 \mathrm{~h} 、 2.5 \mathrm{~h} 、 3 \mathrm{~h} 、 4 \mathrm{~h} 、 5 \mathrm{~h} 、 6 \mathrm{~h}$ 时间段分别取 $1 \mathrm{~mL}$ 溶液并稀释至一定的倍数, 采用原子吸收分光 光度计进行测试。每次取出溶液后, 立刻添加相同 体积、相同 $\mathrm{pH}$ 的磷酸盐缓冲溶液保持总溶液体积 不变, 并根据标准曲线计算 KDF 的含量。按公式(3) 计算包封率，按公式(4)计算载药率:

$$
\begin{aligned}
& \text { 包封率 }=\frac{m_{1}}{m_{2}} \times 100 \% \\
& \text { 载药率 }=\frac{m_{1}}{m_{3}} \times 100 \%
\end{aligned}
$$

式中, $m_{1}$ 为抗菌微球中 $\mathrm{KDF}$ 含量 $(\mathrm{g}), m_{2}$ 为 $\mathrm{KDF}$ 投 加量 $(\mathrm{g}), m_{3}$ 为抗菌微球的质量 $(\mathrm{g})$ 。

\section{6 抗菌微球体外抗菌测试}

采用微孔板法进行抗菌测试。使用 $200 \mathrm{~mL}$ 去 离子水溶解 $2 \mathrm{~g}$ 肤蛋白炼、 $1.0 \mathrm{~g}$ 牛肉膏、 $1.0 \mathrm{~g}$ 氯化
钠, 调节溶液 $\mathrm{pH}$ 为 7.4 , 在高温高压条件下灭菌 $20 \mathrm{~min}$, 得到液体培养液。添加 $\mathrm{CS} / \mathrm{CMC} /$ Zeolite $\mathrm{P} / \mathrm{KDF}$ 抗菌 微球(Zeolite $\mathrm{P}$ 含量为 $1.5 \mathrm{wt} \%$ )配制 $96 \mathrm{mg} / \mathrm{mL}$ 浓度 抗菌母液, 将 $96 \mathrm{mg} / \mathrm{mL}$ 的母液依次稀释成 $48 、 24$ 、 $12 、 6 \mathrm{mg} / \mathrm{mL}$ 。向样品测定孔中加入上述浓度的抗 菌材料培养液和纯液体培养基各 $200 \mu \mathrm{L}$, 每个浓 度重复 3 组实验, 依次加入大肠杆菌菌悬液 $10 \mu \mathrm{L}$ 。在 $37{ }^{\circ} \mathrm{C}$ 恒温振荡培养箱中振荡培养 $24 \mathrm{~h}$ 。 用酶标仪在波长为 $630 \mathrm{~nm}$ 处测定各组吸光度(即 $\mathrm{OD}_{630}$ 值), 计算其抑菌率 ${ }^{[21]}$ 。抑菌率的计算公式 为(5):

$$
\text { 抑菌率 }=\frac{O D_{1}-O D_{2}}{O D_{1}} \times 100 \%
$$

式中, $O D_{1}$ 为阳性对照 $\mathrm{OD}_{630}$ 值; $O D_{2}$ 为样品 $\mathrm{OD}_{630}$ 值。

\section{2 结果与讨论}

\section{1 水凝胶微球组构}

通过解析原材料 CMC、CS、Zeolite P、 $\mathrm{CS} / \mathrm{CMC} /$ Zeolite P 水凝胶微球的红外结果(图 2)显 示, $\mathrm{CMC}$ 和 $\mathrm{CS}$ 在 3453 和 $3440 \mathrm{~cm}^{-1}$ 处的峰分别归 因于 $\mathrm{CMC}$ 中的 $\mathrm{O}-\mathrm{H}$ 和 $\mathrm{CS}$ 中的 $\mathrm{O}-\mathrm{H} 、 \mathrm{~N}-\mathrm{H}$ 伸缩振 动。 $2920 \mathrm{~cm}^{-1}$ 处为常见的 $\mathrm{C}-\mathrm{H}$ 伸缩窄峰。 $\mathrm{CMC}$ 图谱 中显示, 1635 和 $1420 \mathrm{~cm}^{-1}$ 处对应 $\mathrm{COO}^{-}$离子基团的对 称和不对称伸缩振动吸收峰, $1000 \sim 1200 \mathrm{~cm}^{-1}$ 之间的 吸收带对应 $\mathrm{C}-\mathrm{O}$ 键的伸缩振动吸收峰 ${ }^{[22-23]}$ 。 $\mathrm{CS}$ 图 谱显示, 1645、1300、1460 $\mathrm{cm}^{-1}$ 以及 1020 1200 $\mathrm{cm}^{-1}$ 的吸收带, 分别对应-NH 和羰基伸缩振动, 多糖骨架 上的 $\mathrm{C}-\mathrm{N}$ 和 $\mathrm{C}-\mathrm{O}$ 伸缩振动。在 Zeolite $\mathrm{P}$ 的图谱中, $600 \sim 1000 \mathrm{~cm}^{-1}$ 的吸收带分别是分子篮中 $\mathrm{Si}-\mathrm{O}, \mathrm{Al}-\mathrm{O}$ 和 $\mathrm{Si}-\mathrm{O}-\mathrm{Si}$ 骨架振动所致, 证明成功制备了 Zeolite $\mathrm{P}^{[24-25]}$ 。

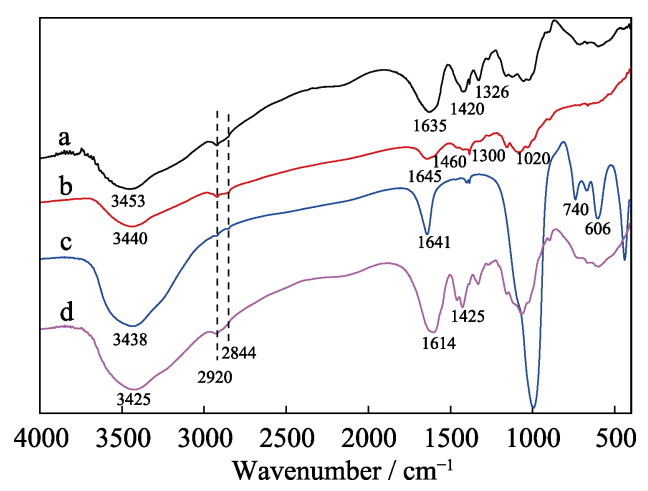

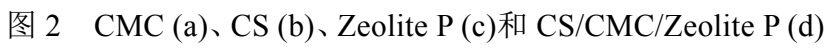
红外光谱图

Fig. 2 FT-IR spectra of CMC (a), CS (b), Zeolite P (c) and $\mathrm{CS} / \mathrm{CMC} /$ Zeolite P (d)

CS: chitosan; CMC: carboxymethyl cellulose; Zeolite P: Zeolite P molecular sieve 
$\mathrm{CS} / \mathrm{CMC} /$ Zeolite P 谱图, 与 CMC、CS 和 Zeolite $\mathrm{P}$ 在 3453、3440 和 $3438 \mathrm{~cm}^{-1}$ 处的峰值相比, 偏移 到 $3425 \mathrm{~cm}^{-1}$, 这是因为 Zeolite P 与 CS、CMC 之间 氢键的作用, 使得振动频率降低。在 1400 1600 $\mathrm{cm}^{-1}$ 区间一些小峰强度增加, 在 $1614 \mathrm{~cm}^{-1}$ 处观察到了 一个新的稍宽吸收带, 这是由于 $\mathrm{CS}$ 的氨基和 $\mathrm{CMC}$ 的羧基相互作用 ${ }^{[26]}$ 。另外, $\mathrm{CS} / \mathrm{CMC} /$ Zeolite $\mathrm{P}$ 与 Zeolite P 相比, 600 1000 $\mathrm{cm}^{-1}$ 的骨架振动特征峰都 存在, 但是强度有所减小, 证实了聚合物中存在 Zeolite $\mathrm{P}$, 这也为人们理解 $\mathrm{CS} / \mathrm{CMC} /$ Zeolite $\mathrm{P} / \mathrm{KDF}$ 抗 菌微球的组构变化提供了理论依据(图 3)。

\section{2 水凝胶微球热稳定性}

图 4 为 $\mathrm{CMC} 、 \mathrm{CS} 、 \mathrm{CS} / \mathrm{CMC}$ 和 $\mathrm{CS} / \mathrm{CMC} /$ Zeolite $\mathrm{P}$ 水凝胶微球的热重分析曲线, 可以看出各个样品降 解过程大致分为三个阶段: 80 200 ${ }^{\circ} \mathrm{C} 、 250 \sim 350{ }^{\circ} \mathrm{C}$ 和 $400 \sim 550{ }^{\circ} \mathrm{C}$, 分别对应 CMC 和 CS 多糖结构中氢键 结合水的分解, 附着在 Zeolite P 上的水分蒸发, 和 $\mathrm{CMC}$ 和 CS 聚合物的降解和分解, 形成碳质残渣。 从失重特征看, $\mathrm{CS} / \mathrm{CMC} /$ Zeolite $\mathrm{P}$ 水凝胶微球在温 度 $\left(\mathrm{T}_{50}\right)$ 直到 $690{ }^{\circ} \mathrm{C}$ 才损失一半重量, 而 $\mathrm{CS} / \mathrm{CMC}$ 、 $\mathrm{CMC} 、 \mathrm{CS}$ 分别在 $488 、 325$ 和 $365{ }^{\circ} \mathrm{C}$ 已经损失一半 重量, 说明 $\mathrm{CS} / \mathrm{CMC}$ 水凝胶微球比天然聚合物具有 更高的热稳定性, 添加 Zeolite P 使复合微球热稳定 性进一步提高。这是由于在聚电解质复合网络结构 中, 有刚性分子篮分布, Zeolite P 与 CS 和 CMC 之 间形成氢键, 以及 $\mathrm{CS}$ 包覆在 $\mathrm{CMC}$ 表面, 羧酸根基 团(CMC) 和氨基 $(\mathrm{Cs})$ 的极性相互作用形成的聚电解 质复合物, 使微球复配结构更稳定, 提高了复合体 系的热稳定性。因此, CS/CMC/Zeolite P 药物传递系 统在生理温度下是稳定的。

\section{3 水凝胶微球微观形貌}

通过 SEM 观察复合水凝胶微球形貌(图 5)。在 制备过程中可以观察到, 干燥前的微球形状近似球 形且表面平滑, 粒径 3 4 $\mathrm{mm}$ 。而干燥后的微球粒径 为 $2 \sim 3 \mathrm{~mm}$, 这是因在干燥过程中, 微球失去水分 后, 聚合物网络部分收缩, 水凝胶微球体积下降。 在图 5 中 $\mathrm{CS} / \mathrm{CMC} /$ Zeolite $\mathrm{P}$ 水凝胶微球内部观察到 Zeolite $\mathrm{P}$ 缠绕或镶嵌在 $\mathrm{CMC}$ 基质中, 结合热稳定 性分析, Zeolite P 的刚性特征对微球骨架具有一定 的支撑作用 ${ }^{[14]}$ 。

\section{4 水凝胶微球溶胀行为}

干燥微球吸水膨胀是由于 $\mathrm{CS}$ 和 $\mathrm{CMC}$ 亲水基团 水合作用, 游离水分子穿透微球聚合物网络中的刚 性孔隙, 溶胀度升高。而 $\mathrm{pH}$ 敏感微球的溶胀机制是 缘于不同结构基团的质子化过程。

形成的微球在 $\mathrm{pH}=1.2$ 的环境下, 溶胀率最低, 是由于 $\mathrm{CS}$ 与 $\mathrm{CMC}$ 之间离子作用增强, 从而形成外 部致密的网状结构, 导致溶胀率较小。酸性条件下, $\mathrm{CMC}$ 会形成致密的凝胶, 导致 $\mathrm{CMC}$ 结构压缩, 抑 制溶胀。 $\mathrm{pH}$ 升高后, 诱导 $\mathrm{CMC}$ 中可电离 $\mathrm{COOH}$ 基 团去质子化, 转换为带负电荷的羧酸根离子, 同时 缓冲溶液中的磷酸根离子置换出 $\mathrm{Ca}^{2+}$, 导致更高的 静电排斥力, 从而增大渗透压, 使更多的水分子渗 入微球中 ${ }^{[27]}$, 造成微球溶胀。

图 6 为不同浓度下 Zeolite $\mathrm{P}$ 的添加量对 $\mathrm{CS} / \mathrm{CMC} /$ Zeolite $\mathrm{P}$ 溶胀行为的影响。在 $\mathrm{pH}=1.2$ 时, $\mathrm{CS} / \mathrm{CMC}$ 水凝胶微球稳定性低, 易溶解破裂, 添加 Zeolite P 之后对微球的骨架结构起到一定的支撑作用, 能够 维持微球形貌稳定、不降解破裂; 在 $\mathrm{pH}=7.4$ 时, 添 加 $0.5 \%$ Zeolite P 的微球具有更高的溶胀率, Zeolite P

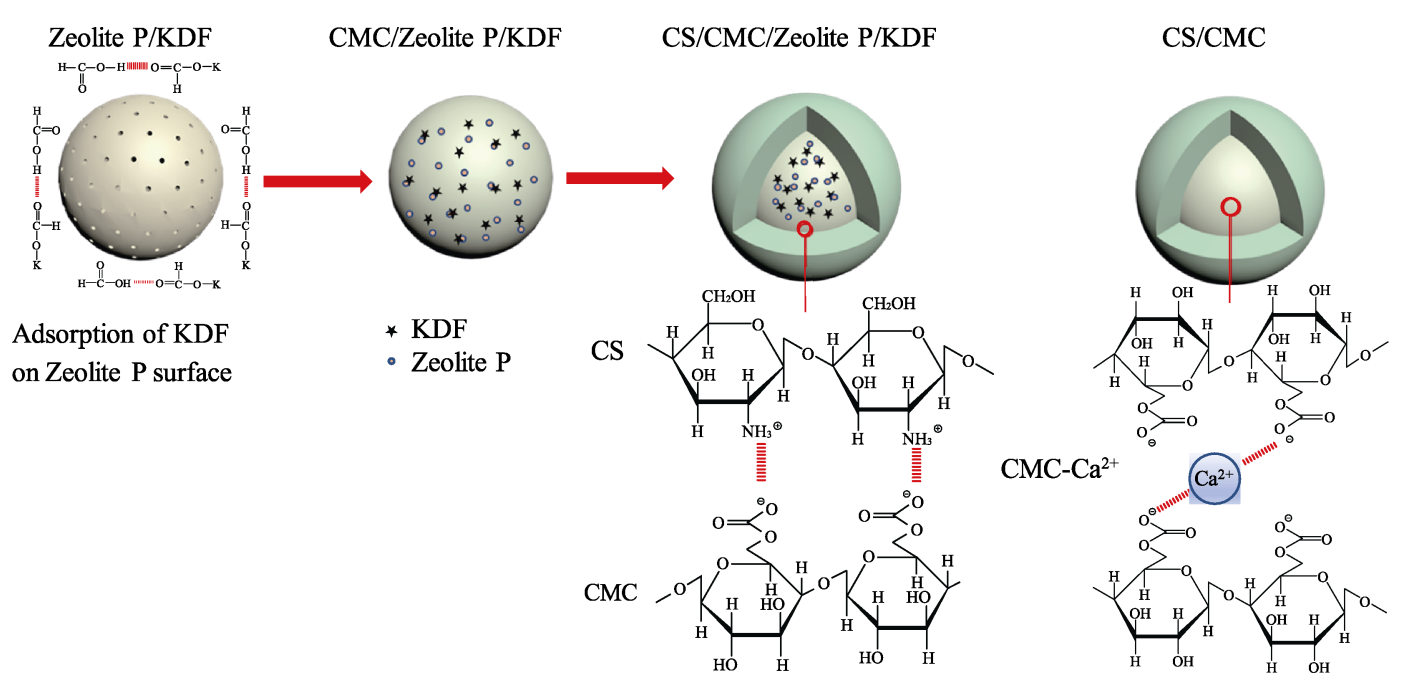

图 $3 \mathrm{CS} / \mathrm{CMC} /$ Zeolite/KDF 微球结构

Fig. 3 Structures of CS/CMC/Zeolite/KDF microspheres 


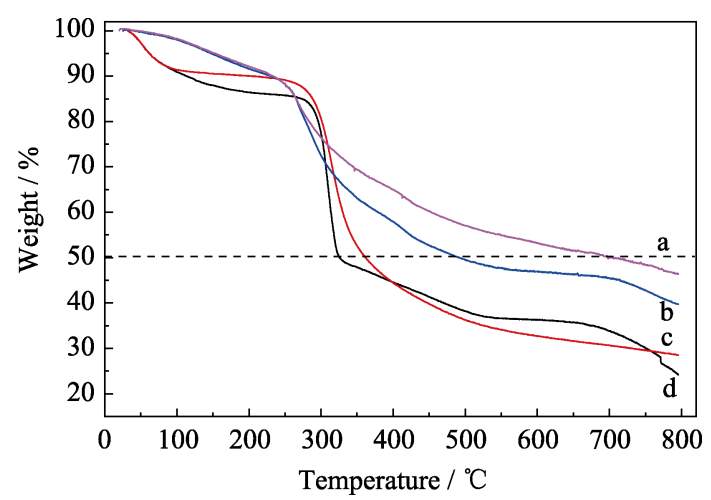

图 $4 \mathrm{CS} / \mathrm{CMC} /$ Zeolite $\mathrm{P}(\mathrm{a}) 、 \mathrm{CS} / \mathrm{CMC}(\mathrm{b}) 、 \mathrm{CMC}(\mathrm{c})$ 和 $\mathrm{CS}(\mathrm{d})$ 的热重分析

Fig. 4 TGA analysis of $\mathrm{CS} / \mathrm{CMC} /$ Zeolite $\mathrm{P}(\mathrm{a}), \mathrm{CS} / \mathrm{CMC}(\mathrm{b})$, $\mathrm{CMC}(\mathrm{c})$ and $\mathrm{CS}(\mathrm{d})$

CS: chitosan; CMC: carboxymethyl cellulose; Zeolite P: Zeolite P molecular sieve
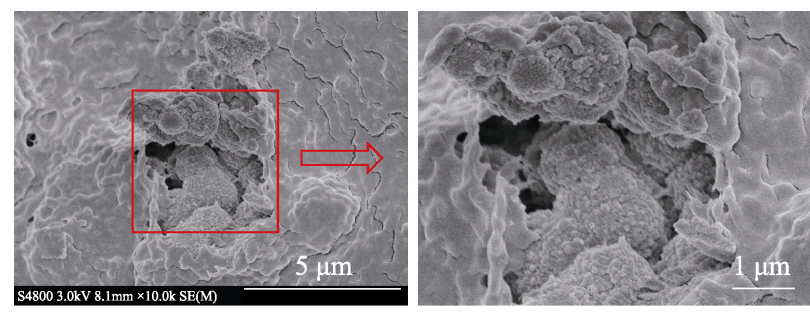

图 $5 \mathrm{CS} / \mathrm{CMC} /$ Zeolite $\mathrm{P}$ 的内部 $\mathrm{SEM}$ 照片

Fig. 5 Internal SEM images of $\mathrm{CS} / \mathrm{CMC} /$ Zeolite $\mathrm{P}$
可以适当扩张水凝胶网络结构，增强 $\mathrm{CMC}$ 基质内 的孔隙和自由空间，因此复合水凝胶中保留了更多 的水分子，从而导致更高的溶胀率。但随着 Zeolite P 的添加量的增加, $\mathrm{CS} / \mathrm{CMC} /$ Zeolite $\mathrm{P}$ 微球溶胀率有 所减小, 这是由于过多的 Zeolite P 镶嵌缠绕在 CMC 三维骨架结构中，限制了 $\mathrm{CMC}$ 链段的扩展，导致微 球的溶胀率降低。说明 $\mathrm{CS} / \mathrm{CMC} /$ Zeolite $\mathrm{P}$ 微球具有 良好的 $\mathrm{pH}$ 敏感性，可以适用胃肠道环境下的药物 递送。

\section{$2.5 \mathrm{KDF}$ 缓释行为}

图 7(a,b)模拟体内胃肠环境，研究不同 Zeolite $\mathrm{P}$ 添加量对 $\mathrm{KDF}$ 释放行为的影响。在酸性环境下吸 水量较少, 可减少 $\mathrm{KDF}$ 溶出释放; 在中性或偏碱性 环境下吸水溶胀, 使 KDF 缓慢溶出。模拟胃液环境 中释放效果明显低于模拟肠道的释放效果, Zeolite $\mathrm{P}$ 的含量越多, $\mathrm{KDF}$ 释放得越慢, 加入 Zeolite $\mathrm{P}$ 可 以越有效地减缓 KDF 的释放。而添加 $1.5 \mathrm{wt} \%$ Zeolite $\mathrm{P}$ 水凝胶微球不仅可有效减缓 KDF 释放, 而 且 $\mathrm{KDF}$ 负载量高、包封率达 $47.75 \%$ 、载药率达 $23.88 \%$ 。

零级释放动力学方程 $M_{t} / M_{\infty}=k_{0} t\left(M_{\infty}\right.$ 和 $M_{t}$ 各自 表示最初的释放体系中的总药物量和 $t$ 时刻微球中 的残留量, $k_{0}$ 表示零级释放常数), 可模拟常见的糖
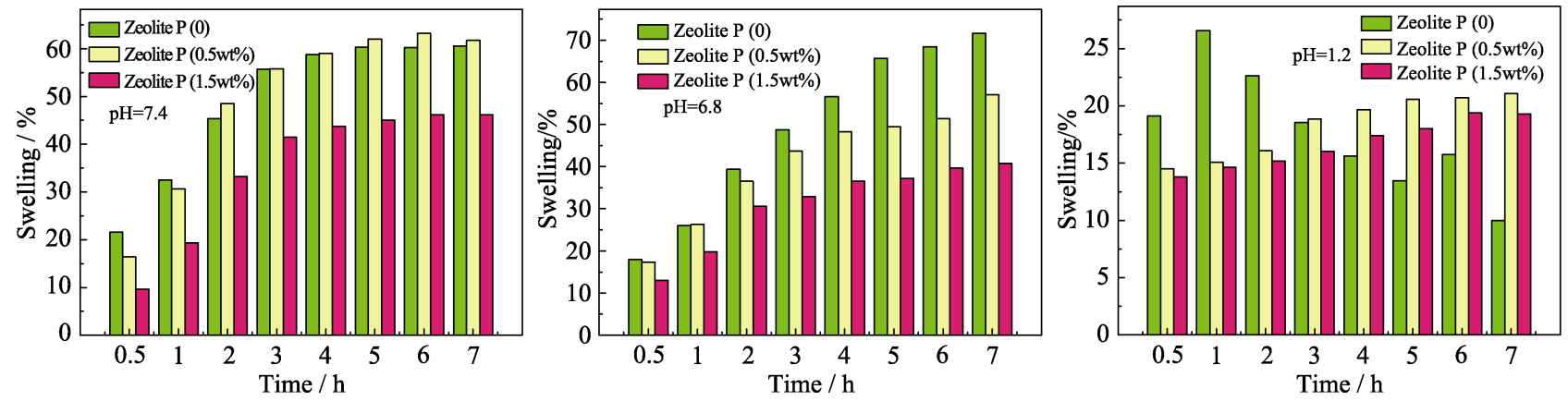

图 6 在不同 $\mathrm{pH}$ 环境下, Zeolite $\mathrm{P}$ 添加量对 $\mathrm{CS} / \mathrm{CMC} /$ Zeolite $\mathrm{P}$ 溶胀行为的影响

Fig. 6 Effect of Zeolite $\mathrm{P}$ concentration on swelling ratios of $\mathrm{CS} / \mathrm{CMC} /$ Zeolite $\mathrm{P}$ in $\mathrm{pH} 7.4$, pH 6.8 and $\mathrm{pH} 1.2$
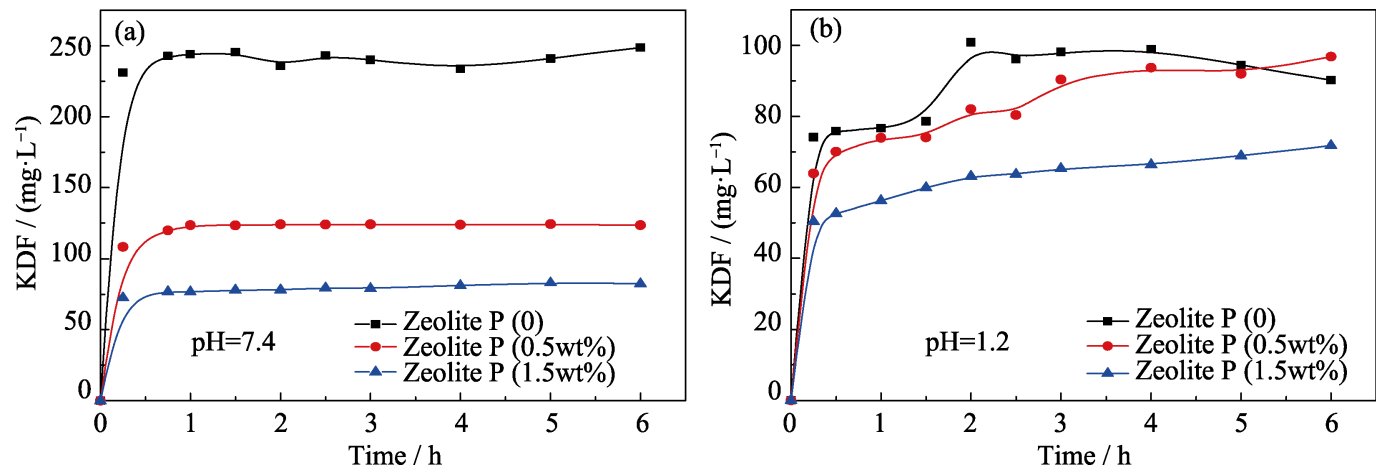

图 7 在不同 $\mathrm{pH}$ 环境下, Zeolite $\mathrm{P}$ 的添加量对 $\mathrm{CS} / \mathrm{CMC} /$ Zeolite $\mathrm{P} / \mathrm{KDF}$ 微球释放 KDF 的行为影响

Fig. 7 Effect of Zeolite P concentration on KDF release profiles of CS/CMC/Zeolite P/KDF in pH 7.4 (a) and pH 1.2 (b) 
衣药物和胶囊药物的释放过程; 一级释放动力学方 程 $M_{t}=M_{\infty}\left(1-\mathrm{e}^{-k t}\right)\left(M_{\infty}\right.$ 表示终点释放的药物总量, $k$ 是 一级释放常数)适用包含水溶性药物的多孔基质; Higuchi 模型动力学方程 $M_{t} / M_{\infty}=k_{\mathrm{H}} t^{1 / 2}\left(M_{t} / M_{\infty}\right.$ 代表当 时释放的药物比例, $k_{\mathrm{H}}$ 是 Higuchi 常数)适合对 $\mathrm{CS} / \mathrm{CMC} /$ Zeolite $\mathrm{P} / \mathrm{KDF}$ 抗菌微球释放动力学的机制 分析 ${ }^{[28-30]}$ 。

本研究分别在模拟胃液(图 8)和肠道(图 9)中对 $\mathrm{CS} / \mathrm{CMC} /$ Zeolite $\mathrm{P} / \mathrm{KDF}$ 的释放动力学进行研究。由 其释放动力学拟合曲线可知, 在胃液中 $\mathrm{CS} / \mathrm{CMC} /$ Zeolite $\mathrm{P} / \mathrm{KDF}$ 相关系数 $R^{2}$ 在零级、一级动力学方程 以及 Higuchi 拟合方程中分别为 $0.37238 、 0.73802$ 和 0.64313 , 在胃液环境中 $\mathrm{KDF}$ 的释放符合一级动力学 方程。在肠道中 $\mathrm{CS} / \mathrm{CMC} /$ Zeolite $\mathrm{P} / \mathrm{KDF}$ 相关系数 $R^{2}$ 在零级、一级动力学方程以及 Higuchi 拟合方程中分 别为 $0.15118 、 0.71701$ 和 0.39859 , 在肠道环境中 KDF 的释放符合一级动力学方程。拟合结果表明制备的抗 菌微球符合水溶性药物在多孔基质中的释放模型。

\section{6 体外抗菌}

大肠杆菌(Escherichia coli)主要在猪的产仔期 至断乳期导致仔猪的胃肠道感染、腹江和生长缓慢。 针对大肠杆菌的抑菌生长实验表明(图 10), 当抗菌
微球浓度在 6 96 mg/mL 范围, 随着浓度的增加对 大肠杆菌的抑制率呈直线上升，最高可以达到 $83 \%$ 。这是因为抗菌微球在有效浓度范围内, 随着 浓度的增大，释放的 $\mathrm{KDF}$ 增多，抑制大肠杆菌生长 和增殖的效果增强。同时进行的 3 个平行实验结果 (图 10)显示: 3 次实验中相同浓度下的抗菌微球对大 肠杆菌的抑菌率差异不大, 说明抗菌微球中的 KDF 是均匀分布的。可见, 制备的 $\mathrm{CS} / \mathrm{CMC} /$ Zeolite $\mathrm{P} / \mathrm{KDF}$ 抗菌微球对大肠杆菌有良好的抑菌效果。

\section{3 结论}

本研究采用凝聚法制备了 $\mathrm{CS} / \mathrm{CMC} /$ Zeolite $\mathrm{P} / \mathrm{KDF}$ 抗菌微球, 解析了 $\mathrm{CS}$ 与 $\mathrm{CMC}$ 通过离子键形 成结构稳定的聚电解质复合物的构建过程, 所引入 的无机刚性 Zeolite P 能够缠绕镶嵌在 CMC 基质中, 有效提高了水凝胶微球的稳定性，使负载在其中的 $\mathrm{KDF}$ 具有较好的缓释性能。

模拟胃肠道 $\mathrm{pH}$ 环境下, KDF 既能在胃中酸性环 境下减少释放，又能在中性或偏碱性肠道环境中缓 慢释放，其释放过程符合一级动力学方程。抗菌液浓 度为 $96 \mathrm{mg} / \mathrm{mL}$ 时对大肠杆菌的抑菌率达到 $83 \%$ 。
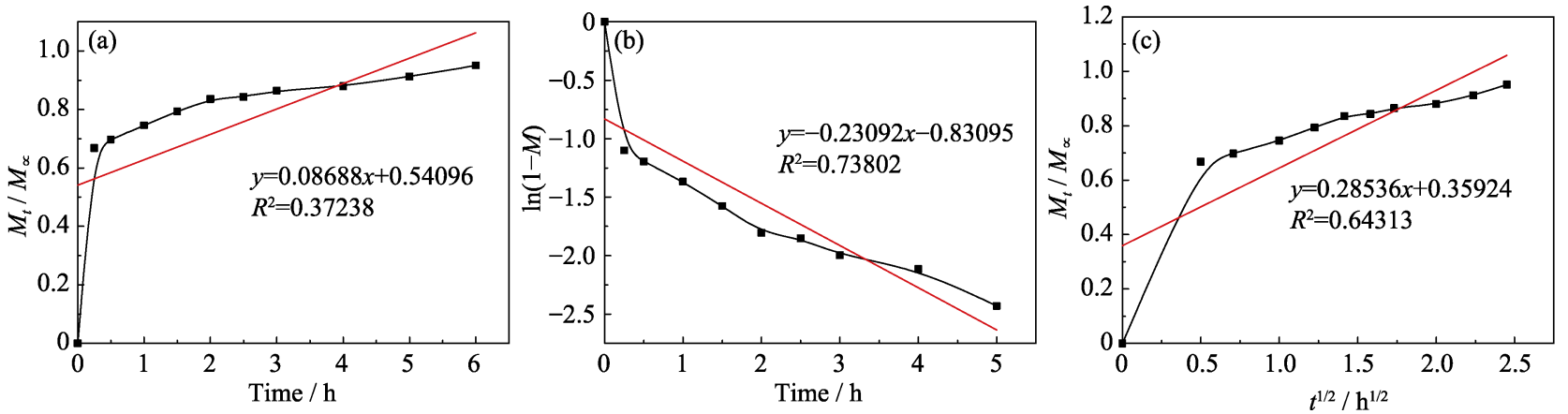

图 $8 \mathrm{CS} / \mathrm{CMC} /$ Zeolite $\mathrm{P} / \mathrm{KDF}$ 在模拟胃液中的释放动力学方程及其零级动力学拟合曲线 $(\mathrm{a})$,

一级动力学拟合曲线(b), Higuchi 方程拟合曲线(c)

Fig. 8 Kinetic equation of $\mathrm{CS} / \mathrm{CMC} /$ Zeolite $\mathrm{P} / \mathrm{KDF}$ in simulated gastric fluid with curves of Zero order kinetics (a)

First order kinetics (b) and Higuchi equations (c)
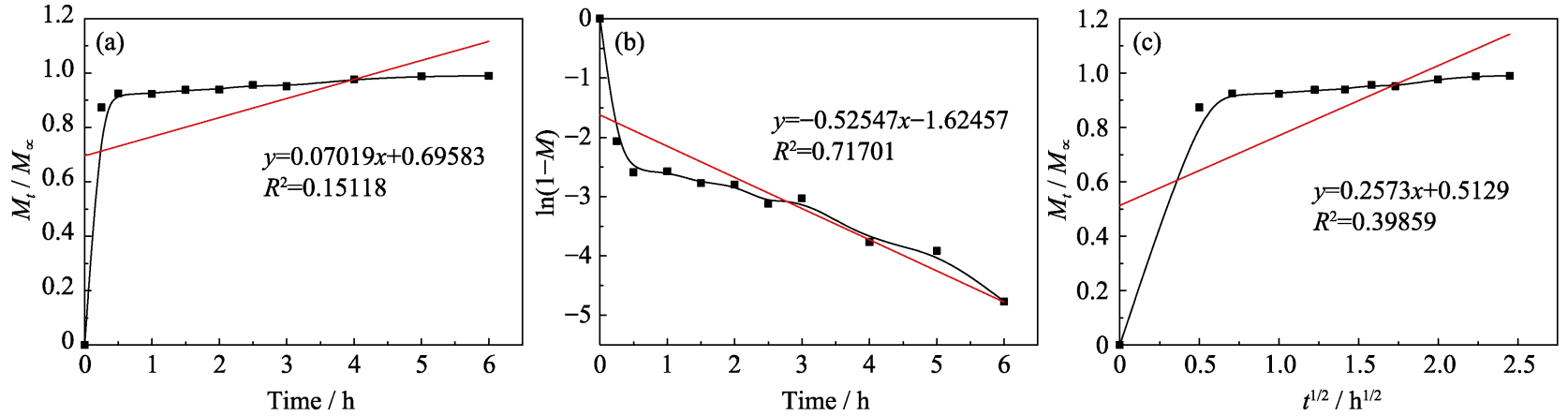

图 $9 \mathrm{CS} / \mathrm{CMC} /$ Zeolite $\mathrm{P} / \mathrm{KDF}$ 在模拟肠道环境中的拟合曲线及其动力学方程

Fig. 9 Kinetic equation of $\mathrm{CS} / \mathrm{CMC} /$ Zeolite $\mathrm{P} / \mathrm{KDF}$ in simulated intestinal fluid with curves of Zero order kinetics (a),

First order kinetics (b), Higuchi equations (c) 


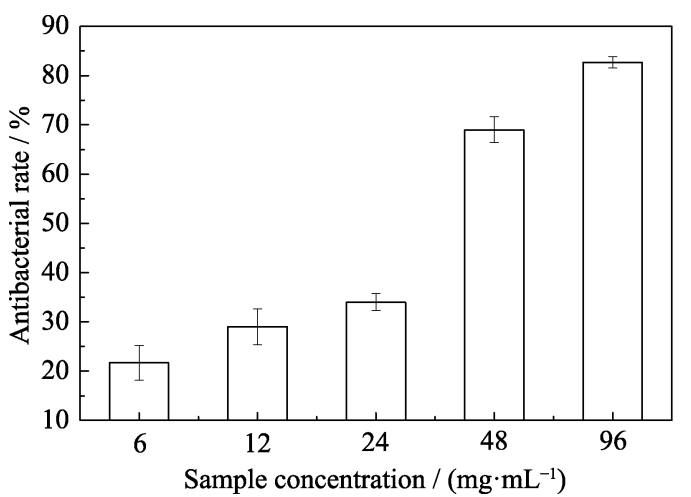

图 10 抗菌液对大肠杆菌的抑菌率

Fig. 10 Antibacterial rates of antibacterial agents with different concentrations on Escherichia coli

\section{参考文献:}

[1] MARQUARDT R R, LI S Z. Antimicrobial resistance in livestock: advances and alternatives to antibiotics. Animal Frontiers, 2018, 8(2): $30-37$.

[2] CANIÇA M, MANAGEIRO V, ABRIOUEL H, et al. Antibiotic resistance in foodborne cacteria. Trends in Food Science \& Technology, 2019, 84: 41-44.

[3] LIN W S, WANG H X, LI Q B, et al. Optimization of green synthesis of potassium diformate and its potential as a mold inhibitor for animal feed. Industrial and Engineering Chemistry Research, 2010, 49(13): 5981-5985.

[4] XIA S S, YAO W, ZOU B, et al. Effects of potassium diformate on the gastric function of weaning piglets. Animal Production Science, 2016, 56: 1161-1166.

[5] CANIBE N, STEIEN S H, OVERLAND M, et al. Effect of $\mathrm{K}$-diformate in starter diets on acidity, microbiota, and the amount of organic acids in the digestive tract of piglets, and on gastric alterations. Journal of Animal Science, 2001, 79(8): 2123-2333.

[6] PAULICKS B R, ROTH F X, KIRCHGESSNER M. Effects of potassium diformate (Formi ${ }^{\circledR}$ LHS) in combination with different grains and energy densities in the feed on growth performance of weaned piglets. Journal of Animal Physiology and Animal Nutrition, 2000, 84(3/4): 102-111.

[7] MROZ Z, REESE D E, OVERLAND M. The effects of potassium diformate and its molecular constituents on the apparent ileal and fecal digestibility and retention of nutrients in growing-finishing pigs. Journal of Animal Science, 2002, 80(3): 681-690.

[8] ZHANG X H, CHEN N C, TANG Q, et al. Slow releasing behaviors of potassium diformate from zeolite A. Journal of the Chinese Ceramic Society, 2013, 41(2): 245-250.

[9] HU Y, GUO X W, PEI Y C, et al. Zeolite X with potassium diformate as a sustained-release antibacterial agent. Bulletin of Materials Science, 2019, 42(2): 66-70.

[10] RASOULZADEH M, NAMAZI H. Carboxymethyl cellulose/ graphene oxide bio-nanocomposite hydrogel beads as anticancer drug carrier agent. Carbohydrate Polymers, 2017, 168: 320-326.

[11] SHARIATINIA Z. Pharmaceutical applications of chitosan. Advances in Colloid and Interface Science, 2019, 263: 131-194.

[12] WANG S J, ZHANG Q, TAN B, et al. pH-sensitive poly(vinyl alcohol)/sodium carboxymethyl cellulose hydrogel beads for drug delivery. Journal of Macromolecular Science, Part B: Physics, 2011, 50(12): 2307-2317.
[13] MITSUMATA T, SUEMITSU Y, FUJII K, et al. pH-response of chitosan, $\kappa$-carrageenan, carboxymethyl cellulose sodium salt complex hydrogels. Polymer, 2003, 44(23): 7103-7111.

[14] ZHU Y L, GU Y, CHEN K P, et al. Research progress in synthesis and application of microcapsules with organic-inorganic hybrid shell. Journal of Functional Materials, 2015, 46(19): 19007-19013.

[15] ZHAI Q Z. Preparation and controlled release of mesoporous MCM-41/propranolol hydrochloride composite drug. Journal of Microencapsulation, 2013, 30(2): 173-180.

[16] ZHANG L, PENG Y X, ZHANG J, et al. Adsorptive and catalytic properties in the removal of volatile organic compounds over zeolite-based materials. Chinese Journal of Catalysis, 2016, 37(6): 800-809.

[17] KHODAVERDI E, HONARMANDI R, ALIBOLANDI M, et al. Evaluation of synthetic zeolites as oral delivery vehicle for antiinflammatory drugs. Iranian Journal of Basic Medical Sciences, 2014, 17(5): 337-343.

[18] GAO S S, CHEN N C, PEI Y C, et al. Hydrothermal reaction conditions for preparation of zeolite $\mathrm{P}$ from natural stilbite as raw material. Journal of Synthetic Crystals, 2018, 47(6): 1096-1101.

[19] ZHANG C Y, CHEN N C, ZHANG X H, et al. In situ preparation and structure of zeolites A, X and P. Journal of the Chinese Ceramic Society, 2014, 42(10): 1332-1336.

[20] ZHANG X H. Study on Preparation of Zeolite Antibacterial Agents and Antibacterial Properties. Guilin: Master's Thesis of Guilin University of Technology, 2013.

[21] JIN X X, WANG J T, BAI J. Synthesis of Schiff base from chitosan and cinnamaldehyde and its antimicrobial activity. Journal of Chemical Engineering of Chinese Universities, 2010, 24(4): 645-650.

[22] YADOLLAHI M, NAMAZI H. Synthesis and characterization of carboxymethyl cellulose/layered double hydroxide nanocomposites. Journal of Nanoparticle Research, 2013, 15(4): 2-9.

[23] YADOLLAHI M, NAMAZI H, BARKHORDARI S. Preparation and properties of carboxymethyl cellulose/layered double hydroxide bionanocomposite films. Carbohydrate Polymers, 2014, 108(1): 83-90.

[24] BEHIN J, KAZEMIAN H, ROHANI S. Sonochemical synthesis of zeolite NaP from clinoptilolite. Ultrasonics Sonochemistry, 2016, 28: $400-408$.

[25] DONG X X, HONG J H, HE G. Synthesis of zeolite NaP from low-grade kaolin with high silica content. Journal of Synthetic Crystals, 2016, 45(16): 2650-2654

[26] WANG R, SHOU D, LÜ O Y, et al. pH-controlled drug delivery with hybrid aerogel of chitosan, carboxymethyl cellulose and graphene oxide as the carrier. International Journal of Biological Macromolecules, 2017, 103: 248-253.

[27] LEE J H, CHOI Y M, PAIK U, et al. The effect of carboxymethyl cellulose swelling on the stability of natural graphite particulates in an aqueous medium for lithium ion battery anodes. Journal of Electroceramics, 2006, 17(2): 657-660.

[28] YU W L, LIU W H, ZHANG L, et al. Preparation of B-vitamins microcapsules and studies on its slow-release kinetics. Journal of Chinese Institute of Food Science and Technology, 2019, 19(12): 122-132.

[29] WANG R, SHOU D, LV O Y, et al. pH-controlled drug delivery with hybrid aerogel of chitosan, carboxymethyl cellulose and graphene oxide as the carrier. International Journal of Biological Macromolecules: Structure, Function and Interactions, 2017, 103: 248-253.

[30] KORSMEYER R W, GURNY R, DOELKER E, et al. Mechanisms of solute release from porous hydrophilic polymers. International Journal of Pharmaceutics, 1983, 15(1): 25-35. 